\title{
Risk Factors of Hypoparathyroidism After Thyroidectomy
}

\author{
Zhou Ru ${ }^{1}$, Wang Maofei ${ }^{1}$, Shahabbas Enwer ${ }^{2}$, Yuan Jianming ${ }^{1}$, , Wu Weize ${ }^{1}$ \\ ${ }^{1}$ Department of General Surgery, Luwan Branch, Ruijin Hospital, School of Medicine, Shanghai Jiao Tong University, Shanghai, China \\ ${ }^{2}$ Department of Preventive Medicine, School of Public Health, Fudan University, Shanghai, China
}

\author{
Email address: \\ yuanjm88@hotmail.com (Yuan Jianming) \\ ${ }^{*}$ Corresponding author
}

\section{To cite this article:}

Zhou Ru, Wang Maofei, Shahabbas Enwer, Yuan Jianming, Wu Weize. Risk Factors of Hypoparathyroidism After Thyroidectomy. American Journal of Clinical and Experimental Medicine. Vol. 8, No. 2, 2020, pp. 20-24. doi: 10.11648/j.ajcem.20200802.14

Received: February 15, 2020; Accepted: April 23, 2020; Published: May 29, 2020

\begin{abstract}
OBJECTIVE] Persistent hypocalcemia caused by long-term hypoparathyroidism can significantly reduce the living quality of patients. The purpose of this study was to investigate possible factors affecting postoperative hypoparathyroidism. [METHODS] This study retrospective collected thyroid surgery cases from January 2015 to December 2017 in Luwan Branch of Ruijin Hospital Affiliated to Medical College of Shanghai Jiao Tong University. Moreover, we analyzed demographic data, operative data and perioperative examination indexes. Using logistic regression analysis to investigate possible factors affecting postoperative transient/long-term hypoparathyroidism. [RESULTS] Totally, 1381 people were collected, 343 male (24.84\%) and 1038 female $(75.16 \%)$, the average age was $47.9 \pm 13.0$ years. Postoperative transient hypoparathyroidism occurred in 456 people (33.02\%), and long-term hypoparathyroidism in 21 people (1.52\%). After multivariate analysis, the main risk factors associated with postoperative transient hypoparathyroidism were gender $(\mathrm{P}<0.001$, OR 0.486$)$, maximum diameter of thyroid resection $(\mathrm{P}$ 0.011, OR 1.192), thyroid resection extent $(\mathrm{P}<0.001$, OR 2.974), lymph node dissection( $\mathrm{P} 0.008$, OR 1.569), surgery duration( $\mathrm{P}$ 0.034, OR 1.005), preoperative serum calcium $(\mathrm{P}<0.001$, OR 0.028$)$. The main risk factors associated with postoperative long-term hypoparathyroidism were thyroid resection extent $(\mathrm{P}<0.003$, OR 5.443), surgery duration ( $\mathrm{P}$ 0.021, OR 0.976), postoperative d1PTH $(\mathrm{P}<0.001$, OR 1.199). [CONCLUSIONS] The risk factors for postoperative transient hypoparathyroidism include gender, the extent of thyroid resection, lymph node dissection, duration of surgery and preoperative serum calcium. The extent of resection and duration of surgery were risk factors for postoperative long-term hypoparathyroidism, and PTH on the early morning after surgery (d1PTH) was lower than normal, which had a predictive effect on long-term hypoparathyroidism.
\end{abstract}

Keywords: Thyroid Surgery, Hypoparathyroidism, Logistic Regression Analysis

\section{Introduction}

Thyroid carcinoma (TC) is the fastest-growing malignant tumor in China, with an annual incidence rate of 7.7/lakh. The radical treatment of TC is thyroid surgery. Although it has a good prognosis, the postoperative complications such as recurrent laryngeal nerve injury, hypoparathyroidism are not negligible. The incidence varied and had been reported as ranging from 1.2 to $40 \%$ [1]. Hypocalcemia is a complication of thyroidectomy, most of which is caused by the decrease of parathyroid hormone $[2,3]$. Symptoms of hypocalcemia may be light or heavy, some of which may exist for a long time or even affect life span [4, 5]. Most of hypoparathyroidism is temporary, but minority patients probably will develop longterm hypoparathyroidism, and the incidence of persistent hypocalcemia is $0 \%$ to $5 \%$ [6]. Long-term hypocalcemia leads to osteoporosis, spasm, which is the main factor affecting postoperative life quality of patients. The purpose of this study was to investigate possible factors affecting postoperative hypoparathyroidism and to provide an objective basis for reducing complications.

\section{Materials and Methods}

\subsection{Design}

A retrospective control study of patient was conducted and collected thyroid surgery cases from January 2015 to December 2017 in Luwan Branch of Ruijin Hospital Affiliated to Medical College of Shanghai Jiao Tong University. The inclusion criteria were patients who unilateral or bilateral 
thyroidectomized or partial thyroidectomized with benign or malignant TC. Main exclusion criteria: 1 . Preoperative patients with hypocalcemia (serum calcium $<2.2 \mathrm{mmol} / \mathrm{L}$ ); 2 . Patients in secondary thyroid surgery; 3. Patients with transient hypocalcemia without postoperative follow-up data; 4. Preoperative patients with renal insufficiency and other factors that were affecting serum calcium and phosphorus metabolism; 5. Patients involved in parathyroid surgery. Totally 1381 people were collected, 343 male $(24.84 \%)$ and 1038 female $(75.16 \%$ ), the average age was 47.8 (range, 15-44 y). All patients with postoperative hypoparathyroidism were divided into two groups according to whether show hypoparathyroidism in the early morning after surgery or six months later, and were compared with those with normal. All data were collected from the electronic medical history system database. Demographic data, operative data and perioperative examination indexes were collected.

As this study was a retrospective study, the informed consent of patients was exempted by the ethics committee.

\subsection{Judgment and Treatment}

Routine preoperative testing with serum calcium, parathyroid hormone, and retest early morning after surgery. Transient hypoparathyroidism was defined as postoperative serum calcium $\leq 2.2 \mathrm{mmol} / \mathrm{L}$ or parathyroid hormone $(\mathrm{PTH}) \leq 7 \mathrm{pg} / \mathrm{ml}$, and the above patients were treated with an oral calcium/vitamin D3 supplementation. Moreover, longterm hypoparathyroidism was defined as the retest of low $\mathrm{PTH}(\leq 7 \mathrm{pg} / \mathrm{ml}) \quad 6$ months after the surgery, or the supplementation of calcium and vitamin D3 was still needed to maintain normal serum calcium $(\geq 2.2 \mathrm{mmol} / \mathrm{L})$ [7].

\subsection{Methods}

SPSS 13.0 (SPSS Inc., Chicago, IL) was used for statistical analysis, and the counting data was presented in the form of a percentage. In the measurement data, the average distribution data are presented in the form of mean \pm standard deviation, while the non-normal distribution data are presented in the form of the median (range). Independent-Samples T-Test was used for measurement data, and Chi-square test or Fisher exact probability method was used for counting data. Logistic multivariate regression analysis used the enter method to assess risk factors for transient/long-term hypoparathyroidism. $\mathrm{P}<0.05$ was considered statistically significant.

\section{Result}

\subsection{Demographic Baseline Characteristics}

Totally 1,381 people were collected, 343 males $(24.84 \%)$ and 1,038 females $(75.16 \%)$, the average age was $47.9 \pm 13.0$ years.

\subsection{Perioperative Data}

The average surgical duration of all patients was $84.1 \pm 35.9$ minutes. Postoperative pathological data were collected from all subjects, including 681 people (49.31\%) of benign disease and 700 people $(50.69 \%)$ of malignant tumours. Among the malignant tumours, 691 people $(50.04 \%)$ of papillary carcinoma, 6 people $(0.004 \%)$ of follicular carcinoma, and 3 people $(0.002 \%)$ of medullary carcinoma. Unilateral resection was performed in 719 people $(52.06 \%)$ and bilateral resection in 662 people $(47.94 \%)$. There were 786 people $(56.92 \%)$ of uncomplicated thyroidectomy and 595 people (43.08\%) of thyroidectomy combined with lymph node dissection.

All patients had an average of $2.40 \pm 0.08 \mathrm{mmol} / \mathrm{L}$ of serum calcium, $35.4 \pm 16.1 \mathrm{pg} / \mathrm{L}$ of parathyroid hormone, in preoperative. $2.28 \pm 0.13 \mathrm{mmol} / \mathrm{L}$ of serum calcium and $17.8 \pm 11.7 \mathrm{pg} / \mathrm{L}$ of parathyroid hormone, early morning after surgery. Postoperative transient hypoparathyroidism occurred in 456 people, with an incidence of $33.02 \%$. Among them, 282 people $(20.42 \%)$ of parathyroid hormone were lower than usual, and 308 people $(22.27 \%)$ of serum calcium were lower than usual. Postoperative long-term hypoparathyroidism occurred in 21 cases, with an incidence of $1.52 \%$. Among them, 19 people $(90.48 \%)$ of parathyroid hormone were lower than usual, and 2 people $(9.52 \%)$ of normal serum calcium maintained by calcium supplement.

Baseline data and operative and perioperative information showed in table 1.

Table 1. Univariate analysis of transient hypoparathyroidism.

\begin{tabular}{|c|c|c|c|c|}
\hline Factor & $\begin{array}{l}\text { Transient hypoparathyroidism group } \\
(\mathrm{n}=456)\end{array}$ & $\begin{array}{l}\text { Normal thyroid function group } \\
(\mathrm{n}=925)\end{array}$ & $X^{2} / t$ & $\mathbf{P}$ \\
\hline \multicolumn{5}{|l|}{ Gender } \\
\hline Male (case) & 76 & 267 & \multirow{2}{*}{15.889} & \multirow{2}{*}{$<0.001$} \\
\hline Female (case) & 380 & 658 & & \\
\hline Age (year) & $48.3 \pm 12.9$ & $47.7 \pm 13.0$ & -0.662 & 0.508 \\
\hline Surgery duration $(\mathrm{min})$ & $92.0 \pm 36.6$ & $80.2 \pm 34.6$ & -4.805 & $<0.001$ \\
\hline \multicolumn{5}{|l|}{ Surgical extent } \\
\hline Unilateral (case) & 148 & 571 & \multirow{3}{*}{68.728} & \multirow[t]{2}{*}{$<0.001$} \\
\hline Bilateral (case) & 308 & 354 & & \\
\hline \multicolumn{4}{|l|}{ Lymph node dissection } & \\
\hline Yes (case) & 216 & 570 & \multirow{2}{*}{16.792} & \multirow{2}{*}{$<0.001$} \\
\hline No (case) & 240 & 355 & & \\
\hline Maximum tumor diameter (mm) & $17.7 \pm 12.8$ & $18.7 \pm 14.1$ & 1.046 & 0.296 \\
\hline Maximum diameter of thyroid resection $(\mathrm{cm})$ & $4.79 \pm 1.32$ & $4.51 \pm 1.12$ & -3.397 & 0.001 \\
\hline Preoperative serum calcium (mmol/L) & $2.39 \pm 0.08$ & $2.41 \pm 0.08$ & 3.810 & $<0.001$ \\
\hline Preoperative PTH (ng/L) & $35.5 \pm 17.6$ & $35.3 \pm 15.4$ & -0.141 & 0.888 \\
\hline
\end{tabular}




\subsection{Logistic Multivariate Regression Analysis}

Multivariate analysis showed that gender, thyroid resection extent, lymph node dissection, duration of surgery, the maximum diameter of thyroid resection, and preoperative serum calcium might be associated with transient hypoparathyroidism $(\mathrm{P}<0.05)$. The OR value of gender $($ male $)$ was 0.486 , and the $95 \% \mathrm{CI}$ was $(0.335,0.705)$. The OR value of thyroid resection extent was 2.974 , and the $95 \% \mathrm{CI}$ was $(2.155,4.103)$. The OR value of lymph node dissection was 1.569 , and the $95 \%$ CI was $(1.122,2.194)$. The OR value of the surgery duration was 1.005 , and the $95 \% \mathrm{CI}$ was $(1.000,1.009)$. The OR value of the maximum diameter of thyroid resection was 1.192 , and the $95 \% \mathrm{CI}$ was $(1.041,1.364)$. The OR value of preoperative serum calcium was 0.028 , and $95 \%$ CI was $(0.004,0.203)$. (Table 2$)$

The extent of thyroid resection, surgery duration and postoperative d1PTH may be associated with long-term hypoparathyroidism $(\mathrm{P}<0.05)$. The OR value of the extent of thyroid resection was 5.443 , and the $95 \% \mathrm{CI}$ was $(1.805,16.418)$. The OR value of surgery duration was 0.976 , and the $95 \% \mathrm{CI}$ was $(0.956,0.996)$. The OR value of postoperative d1PTH was 1.199 , and the $95 \% \mathrm{CI}$ was $(1.086,1.324)$. (Table 3$)$

Table 2. Multivariate Logistic regression analysis of transient hypoparathyroidism.

\begin{tabular}{lll}
\hline Factor & $\boldsymbol{P}$ & OR (95\%CI) \\
\hline Gender (male) & $<0.001$ & $0.486(0.335,0.705)$ \\
Age (year) & 0.881 & $1.001(0.989,1.013)$ \\
Thyroid resection extent (bilateral) & $<0.001$ & $2.974(2.155,4.103)$ \\
Lymph node dissection (yes) & 0.008 & $1.569(1.122,2.194)$ \\
Surgery duration (min) & 0.034 & $1.005(1.000,1.009)$ \\
Maximum tumor diameter (mm) & 0.139 & $0.991(0.978,1.003)$ \\
Maximum diameter of thyroid (cm) & 0.011 & $1.192(1.041,1.364)$ \\
Preoperative serum calcium (mmol/L) & $<0.001$ & $0.028(0.004,0.203)$ \\
Preoperative PTH (ng/L) & 0.382 & $0.996(0.987,1.005)$ \\
\hline
\end{tabular}

Table 3. Multivariate Logistic regression analysis of long-term hypoparathyroidism.

\begin{tabular}{lll}
\hline Factor & $\boldsymbol{P}$ & OR (95\%CI) \\
\hline Gender (male) & 0.568 & $0.717(0.229,2.246)$ \\
Age (year) & 0.469 & $0.986(0.950,1.024)$ \\
Thyroid resection extent (bilateral) & 0.003 & $5.443(1.805,16.418)$ \\
Lymph node dissection (yes) & 0.361 & $1.594(0.586,4.334)$ \\
Surgery duration (min) & 0.021 & $0.976(0.956,0.996)$ \\
Maximum tumor diameter (mm) & 0.076 & $0.956(0.909,1.005)$ \\
Maximum diameter of thyroid resection (cm) & 0.332 & $1.254(0.794,1.981)$ \\
Preoperative serum calcium (mmol/L) & 0.816 & $0.481(0.001,228.086)$ \\
Preoperative PTH (ng/L) & 0.060 & $0.965(0.930,1.001)$ \\
Postoperative d1 serum calcium (mmol/L) & 0.376 & $4.376(0.167,114.361)$ \\
Postoperative d1PTH (ng/L) & $<0.01$ & $1.199(1.086,1.324)$ \\
\hline
\end{tabular}

\section{Discussion}

Hypoparathyroidism is one of the most common complications after thyroid surgery. Common causes include the effect of surgery on the blood supply to the parathyroid glands, or direct damage to the parathyroid glands during surgery, and mis-division of the parathyroid glands. In this study, the incidence of postoperative transient hypoparathyroidism was $33.02 \%$, and the incidence of longterm hypoparathyroidism was $1.52 \%$. This study included 1381 cases, after multiple logistic regression analysis, obtained the following conclusion: gender, thyroid resection extent, lymph node dissection, surgery duration, the maximum diameter of thyroid resection, Preoperative serum calcium was associated with transient hypoparathyroidism. Surgery duration, postoperative D1PTH associated with long-term hypoparathyroidism. There was no correlation between age, tumor size and the occurrence of hypoparathyroidism.
Related research shows that the blood supply of parathyroid is mostly from the arterial blood supply of thyroid [8]. Ligation thyroid artery blood supply in thyroid surgery(bilateral) is bound to affect the blood supply of the parathyroid. In this study, both postoperative transient hypoparathyroidism and long-term hypoparathyroidism were more related to bilateral thyroid resection than unilateral thyroid resection. So, for some benign thyroid diseases, the extent of surgery should be narrowed as far as possible to achieve the purpose of protecting the parathyroid function.

Lymph node dissection is often required for malignant TC, and relevant literature had also shown that lymph node dissection in the central group could significantly increase the direct intraoperative damage to the parathyroid or blood supply disorders [9]. Therefore, there is a controversy in the relevant literature at home and abroad about whether to prophylactic neck lymph node dissection for patients who have not been proved to have positive lymph nodes or not. Some scholars believe that prophylactic neck lymph node dissection cannot significantly reduce the probability of 
recurrence and metastasis, but increase the risk of parathyroid injury. In this case study, lymph node dissection was not a risk factor for long-term hypoparathyroidism, which was similar to the results of some national studies [10]. Therefore, standardized lymph node dissection and the negative coloration effect of nano-carbon and other reagents on parathyroid can significantly reduce the mis-incision of parathyroid during lymph node dissection [11].

For the size of the resection of the thyroid itself is one of the influence factors of transient serum calcium. As a result, the author thinks that the volume of the thyroid will mainly cause the reduction of the operative field and the identity of the gland, increased difficulty of protection. Moreover, patients are increasingly demanding about the size of the incision, and the large thyroid increases the difficulty of exposure under the small incision. Preoperative serum calcium was taken as a numerical variable in the calculation process, and its value had a direct influence on the determination of postoperative hypocalcemia.

Surgery duration also affects postoperative transient/longterm hypoparathyroidism in this study. The author believes that the scope, difficulty and degree of precision of the operation will be directly or indirectly reflected in the operation time. However, the control of the duration of surgery is also related to the surgeon's experience, surgical style and other factors, the number of long-term hypocalcemia cases in this study is less ( 21 cases). In this statistical case, the surgeon is experienced in surgery so that this conclusion may be reached, but further proof is needed.

Postoperative D1PTH is meaningful in the long-term hypothyroidism, which can be used as a predictor of longterm hypoparathyroidism [12]. For patients with D1PTH less than usual, calcium supplementation/vitamin D3 should be strengthened. If neglected, failed to get enough calcium supplement or close follow-up review, may weaken parathyroid function recovery [13].

In his study, there were still some limitations regarding longterm hypoparathyroidism: 1 . This study adopts a retrospective, single-centre study design, which may have selection bias in patient selection. 2 . In this study, only 21 people with longterm hypoparathyroidism were collected. Compared with patients who without hypoparathyroidism, the number of cases was relatively small, which may affect the statistical efficacy. On the other hand, no long-term detection results were collected, which may affect the presentation of long-term prognosis. 3. In terms of research statistics, some missing data adopted the method of mean value supplement, which may affect the accuracy of statistical results. However, significant risk factors were generally consistent with previous literature. This study aimed to find out more specific, sensitive and straightforward independent risk factors from the relationship between clinical manifestations, laboratory indicators and persistent severe hypocalcemia, to predict the occurrence of hypoparathyroidism and timely intervention to alleviate the discomfort symptoms of patients. We are currently collecting more case data and applying these independent factors to clinical validation.

\section{Conclusion}

Hypoparathyroidism is common complications after thyroid surgery, and the vast majority of them are transient. Gender, the extent of thyroid resection, lymph node dissection, duration of surgery and preoperative serum calcium are risk factors for postoperative transient hypoparathyroidism. A few of them were turned to long-term hypoparathyroidism. The extent of resection and duration of surgery were risk factors for postoperative long-term hypoparathyroidism, and PTH on the early morning after surgery was lower than usual, which had a predictive effect on long-term hypoparathyroidism.

\section{Declarations}

\section{Ethics Approval and Consent to Participate}

Patient consent was obtained with each patient enrolled in the database. These patients were contacted by telephone to obtain verbal informed consent again before this submission.

\section{Consent for Publication}

All patient information is de-identified.

\section{Availability of Data and Materials}

All data generated or analyzed during this study are included in this published article. The detail datasets used or analyzed during the current study are available from the corresponding author on reasonable request.

\section{Competing Interests}

The authors declare that they have no competing interests.

\section{Funding}

None

\section{Authors' Contributions}

All authors have approved this final article. ZR, WMF, and YJM had full access to all the data in the study and take responsibility for the integrity of the data and the accuracy of the data analysis. YJM and WWZ contributed to the concept and design. YJM and WMF contributed to the acquisition, analysis, or interpretation of data. ZR, WMF and SE drafted the manuscript. All authors contributed to the critical revision of the manuscript for relevant intellectual content. YJM and WMF contributed to the statistical analysis.

\section{Acknowledgements}

Dr. $\mathrm{Wu}$ supervised this entire project with the remaining acknowledgments noted above. 


\section{References}

[1] Dionigi G, Baeuzzi A, Bertocchi V, et al . Prospective and surgical usefulness of perioperative parathyroid hormone assay in thyroid surgery [J]. Expert Rev Med Devices, 2008, 5 (6): 699-704.

[2] Dedivitis RA, Aires FT, Cernea CR. Hypoparathyroidism after thyroidectomy: prevention, assessment and management [R]. Curr Opin Otolaryngol Head Neck Surg, 2017, 25 (2): 142-146.

[3] Coimbra C, Monteiro F, Oliveira P, et al. Hypoparathyroidism following thyroidectomy: Predictive factors [J]. Acta Otorrinolaringol Esp. 2017; 68 (2): 106-111.

[4] Caglià P, Puglisi S, Buffone A, et al. Post-thyroidectomy hypoparathyroidism, what should we keep in mind? [R]. Ann Ital Chir, 2017, 6: 371-381.

[5] Almquist M, Ivarsson K, Nordenström E, et al. Mortality in patients with permanent hypoparathyroidism after total thyroidectomy [J]. Br J Surg. 2018; 105 (10): 1313-1318.

[6] Toniato A, BoschinI M, Piotto A, et al. Thyroidectoma and para-thyroid hormone : tracing Hypocalcemia-prone [J]. Am J Surg, 2008, 196 (2): 285-288.
[7] Shoback D. Clinical practice. Hypoparathyroidism [J]. N Engl J Med, 2008, 359 (4): 391-403.

[8] Li Wenjie, XuHaiqian, ZhaiLibin. Parathyroid Micro Vascular Anatomy and Thyroid Lobectomy with Capsular Technique [J]. Chin J Bases Clin General Surg, 2013, 20 (01): 104-107.

[9] Landry CS, Grubbs EG, Hernandez M, et al. Predictable criteria for selective, rather than routine, calcium supplementation following thyroidectomy [J]. Archives of Surgery, 2012, 147 (4): 338.

[10] LvChunhui, Chen Haizhen, ShenXiaohui, et al. Risk factors for hypoparathyroidism after total thyroidectomy [J]. Chin J Gen Surg, 2017, 32 (10): 863-867.

[11] Yin Fengyan, Xing Hao, Zhang Xiaojian, et al. Protective effects of nano-carbon on parathyroid in thyroid surgery [J]. Chin J EndocrSurg, 2015 (2): 144-146.

[12] BaoHaidong, Gong Peng. Risk factors, diagnosis and treatment of hypoparathyroidism after total thyroidectomy [J]. International Journal of Surgery, 2017, 44 (2): 81-84.

[13] Kazaure HS, Sosa JA. Surgical Hypoparathyroidism [R]. Endocrinol Metab Clin North Am, 2018, 47 (4): 783-796. 